\title{
Fisioterapia en la inhibición muscular artrogénica. Revisión de literatura
}

\section{Physiotherapy in arthrogenic muscle inhibition: literature review}

\author{
Iván Darío Pinzón Ríos \\ M.Sc. Ciencias de la Actividad Física y Deporte, Especialista en Pedagogía Universitaria, Fisioterapeuta. Universidad Manuela Beltrán \\ Bucaramanga. ivandpr@hotmail.com
}

Fecha de recepción: 30/11/2018

Fecha de corrección 05/20/2019

Fecha de aceptación: 30/06/2019

Fecha de publicación 30/09/2019

\section{Resumen}

El objetivo de este estudio fue investigar la efectividad de la Fisioterapia en la inhibición muscular artrogénica. Se realizaron búsquedas en las bases PEDro, Embase, Scielo y PubMed para identificar ensayos controlados aleatorios (ECA) de intervenciones de Fisioterapia en la inhibición muscular artrogénica. La literatura incluyo referencias en inglés, español y portugués, publicados entre Enero de 2000 y Junio de 2017. Se identificaron 7 ensayos clínicos que se incluyeron en esta revisión y 4 Revisiones Sistemáticas/Meta-análisis. Se realizó una evaluación crítica de la literatura y se procesó una descripción narrativa debido al pequeño número de ECA. La evidencia muestra que la intervención fisioterapéutica puede ser beneficiosa para el manejo de la inhibición muscular artrogénica, sin embargo, el resultado de baja calidad de la evaluación crítica y el pequeño número de ensayos sugieren que se requieren ECA de alta calidad para examinar más a fondo los efectos de la intervención fisioterapéutica en el control de la inhibición muscular artrogénica.

Palabras clave: inhibición muscular, debilidad, tratamiento, terapia física.

\section{Abstract}

The aim of this study was to investigate the effectiveness of physiotherapy in arthrogenic muscle inhibition. We searched the PEDro, Embase, Scielo and PubMed databases to identify randomized controlled trials (RCTs) of physiotherapy interventions in arthrogenic muscle inhibition. The literature included references in English, Spanish and Portuguese, published between January 2000 and June 2017. We identified 7 clinical trials and 4 Systematic Review/Meta-analysis that were included in this review. A critical evaluation of the literature was made and a narrative description was processed due to the small number of RCTs. Evidence shows that physiotherapeutic intervention may be beneficial for the management of arthrogenic muscle inhibition, however, the low quality result of the critical evaluation and the small number of trials suggest that high quality RCTs are required to further examine the effects of physiotherapeutic intervention in the control of arthrogenic muscle inhibition.

Key words: muscle inhibition, weakness, treatment, physical therapy.

\section{Introducción}

En la actualidad, las lesiones de origen músculoesquelético se consideran un problema de Salud Pública, siendo causa frecuente de consulta fisioterapéutica ocupando el $80 \%$ de los pacientes que asisten a este servicio [1]. Desde esta óptica, la inhibición continua de los músculos periarticulares, secundaria a un daño o patología articular, se evidencia en lesiones de las articulaciones que cursan con inflamación o dolor como daños del ligamento cruzado anterior, los esguinces y patologías articulares como artritis reumatoide y osteoartritis, entre otras [2]. El origen de la inhibición puede variar, pues en patologías inflamatorias y/o con edema articular, se aumenta la descarga de los mecanorreceptores de la articulación afectada, que activan interneuronas inhibitorias Ib de la musculatura periarticular; por otro lado, en lesiones estructurales de los receptores articulares (como la osteoartritis o en lesiones traumáticas) se disminuye la descarga aferente de estos, lo cual disminuye la transmisión aferente la y la actividad muscular [3].

Por eso, antes que se instaure el dolor en una articulación por cualquier causa (traumatismos, enfermedades y/o inducidos en laboratorio), se suceden alteraciones musculares que dan lugar a compromisos biomecánicos, del control motor y del movimiento. Este fenómeno es la inhibición muscular artrogénica (IMA), siendo un mecanismo de defensa donde el sistema nervioso central (SNC) actúa cuando se ha producido una lesión articular, inhabilitando los músculos agonistas que la mueven, conllevándolos 
a deficiencias en la fuerza y atrofia [4,5]. En patologías dolorosas de origen músculoesquelético, se pone de manifiesto una gran vulnerabilidad de la articulación, haciéndola propensa a impactos y movimientos lesivos. Para evitar un daño mayor, el SNC anula la actividad en los grupos musculares periarticulares y al mismo tiempo la articulación envía una descarga anormal aferente hacia los centros nerviosos superiores, alterando la excitabilidad de los músculos que la rodean, desactivando o inhibiendo las motoneuronas a del músculo agonista [4].

Todo este circuito está modulado por el dolor, el cual es definido como "una experiencia sensorial y emocional desagradable asociada con una lesión hística real o potencial" [6] y su percepción incluye una intrincada red neuronal que responden a estímulos tisulares influidos por múltiples factores [7] que modifican la actividad funcional que afecta una determinada articulación, imposibilitando su movimiento. El compromiso articular comúnmente resulta en debilidad de los músculos asociados y los esfuerzos para restaurar la fuerza a menudo no tiene el éxito esperado, incluso en ausencia de dolor [5]. En muchos casos la IMA entorpece la rehabilitación después de una lesión articular, como por ejemplo en la rodilla, al evitar la activación funcional del cuádriceps. Se ha atribuido actividad refleja neuronal en la que la entrada aferente alterada que se origina en la articulación lesionada produce una disminución del impulso motor eferente hacia los músculos, como en el caso del cuádriceps [8,9], por ende, los protocolos de rehabilitación después de la lesión de la rodilla deben centrarse en las alteraciones neuromusculares y mecánicas que se producen como resultado del daño articular [10].

Por tal razón, en el siguiente artículo, analiza la efectividad de la Fisioterapia en la IMA, propendiendo por intervenciones efectivas que mejoran la capacidad funcional y calidad de vida de los pacientes/clientes a quienes se dirigen, mejorando el movimiento corporal humano $(\mathrm{MCH})$ como eje central de estudio del fisioterapeuta. Se realizaron búsquedas en cuatro bases de datos electrónicas, PEDro, Embase, Scielo y PubMed, para identificar ensayos clínicos controlados en inglés, portugués y español, publicados entre Enero de 2000 y Junio de 2017, que mencionaran la efectividad de la fisioterapia en la IMA, sin restricción de edad o género. Los términos de búsqueda fueron arthrogenic muscle inhibition y physical therapy. Todos los estudios potencialmente elegibles fueron combinados con el operador booleano AND, posteriormente se combinaron con el operador booleano OR para omitir duplicados. También se adicionaron 4 revisiones sistemáticas/meta-análisis, así como otras publicaciones impresas que permitieron ampliar el marco conceptual

\section{Resultados}

Tabla 1. Literatura revisada

\begin{tabular}{|c|c|c|c|c|}
\hline $\begin{array}{l}\text { Autor } \\
\text { (año) }\end{array}$ & $\begin{array}{l}\text { Poblaci } \\
\text { ón (n) } \\
\text { edad }\end{array}$ & Grupo (s) & Mediciones & Resultados \\
\hline $\begin{array}{l}\text { Bade et al. } \\
\text { (2017) } \\
{[12]}\end{array}$ & $\begin{array}{c}n=162 \\
63 \pm 7 \\
\text { años }\end{array}$ & $\begin{array}{l}\text { Dos grupos: LI (baja } \\
\text { intensidad) y HI } \\
\text { (alta intensidad) } \\
\text { ambos con uso de } \\
\text { ejercicios de } \\
\text { resistencia } \\
\text { progresiva con } \\
\text { progresión rápida a } \\
\text { ejercicios y } \\
\text { actividades con } \\
\text { soporte de peso. } \\
\text { Ambos grupos } \\
\text { fueron tratados de } \\
\text { forma ambulatoria } \\
\text { de 2-3 veces por } \\
\text { semana durante } 11 \\
\text { semanas (26 } \\
\text { sesiones en total). }\end{array}$ & $\begin{array}{l}\text { Prueba de escalada (SCT, } \\
\text { resultado primario), } \\
\text { prueba de tiempo de } \\
\text { subida y vuelta (TUG), } \\
\text { Caminata de } 6 \text { minutos } \\
\text { (6MW), Índice de } \\
\text { osteoartritis Western } \\
\text { Ontario y McMaster } \\
\text { Universities (WOMAC), } \\
\text { Versión corta de health } \\
\text { survey (SF-12), rango de } \\
\text { movimiento de la rodilla } \\
\text { (ROM), fuerza de los } \\
\text { músculos isquiotibiales, y } \\
\text { activación del cuádriceps. }\end{array}$ & $\begin{array}{l}\text { Ambas intervenciones } \mathrm{HI} \text { y } \mathrm{LI} \text { fueron efectivas para } \\
\text { mejorar la fuerza y la función después de la cirugía de } \\
\text { reemplazo de rodilla. }\end{array}$ \\
\hline $\begin{array}{l}\text { Walsh et } \\
\text { al. } \\
(2016) \\
{[13]}\end{array}$ & $\begin{array}{l}n=22 \\
63 \pm 7 \\
\text { años }\end{array}$ & $\begin{array}{l}\text { Tres Grupos: MWM } \\
\text { (movilización } \\
\text { caudal con } \\
\text { movimiento), } \\
\text { SMWM (auto- } \\
\text { movilización caudal }\end{array}$ & $\begin{array}{l}\text { Las medidas de resultado } \\
\text { primarias incluyeron la } \\
\text { prueba de rotación interna } \\
\text { funcional (FIRST) para la } \\
\text { cadera y la prueba de } \\
\text { rotación interna de asiento }\end{array}$ & $\begin{array}{l}\text { Se realizó un análisis de varianza de dos vías (ANOVA), } \\
\text { interacción grupo } \times \text { tiempo. El ANOVA reveló que la } \\
\text { única mejoría significativa fue en el grupo de MWM para } \\
\text { el FIRT ( } p=0.01 \text { ), en el grupo de control. Los sujetos con } \\
\text { IR reducida de la cadera que reciben una sesión única de } \\
\text { MWM exhibieron IR funcional significativamente } \\
\text { mejorada de su cadera que el grupo de control. }\end{array}$ \\
\hline
\end{tabular}




\begin{tabular}{|c|c|c|c|c|}
\hline & & $\begin{array}{l}\text { con movimiento y } \\
\text { GR (grupo control). }\end{array}$ & $\begin{array}{l}\text { pasivo (DIRT) para la } \\
\text { cadera. }\end{array}$ & \\
\hline $\begin{array}{l}\text { Cho et al. } \\
\text { (2013) } \\
{[14]}\end{array}$ & $\begin{array}{l}n=12 \\
21.5 \pm \\
1.6 \\
\text { años }\end{array}$ & $\begin{array}{l}\text { Un grupo con } \\
\text { medición pre y post } \\
\text { intervención. }\end{array}$ & $\begin{array}{l}\text { EMG: pico del reflejo } \mathrm{H} \text { y la } \\
\text { onda } \mathrm{M} \text { del Sóleo y el } \\
\text { Peronéo largo y la onda } \mathrm{V} \\
\text { del Sóleo solamente. }\end{array}$ & $\begin{array}{l}\text { Aumento significativo en la relación } \mathrm{H} / \mathrm{M} \text { en el sóleo } \\
\text { después de taping de la reubicación fibular en } \\
\text { comparación con el valor inicial, pero no después de la } \\
\text { intervención. } \\
\text { No se observó ninguna diferencia en la relación Peronéo } \\
\text { largo } \mathrm{H} / \mathrm{M} \text { o Sóleo } \mathrm{V} / \mathrm{M} \text { en ninguna sesión. }\end{array}$ \\
\hline $\begin{array}{l}\text { Warner et } \\
\text { al. } \\
\text { (2013) } \\
\text { [15] }\end{array}$ & $\begin{array}{l}n=12 \\
25.6 \pm \\
7.7 \\
\text { años }\end{array}$ & $\begin{array}{l}\text { Tres grupos: } \\
\text { Tratamientos } \\
\text { durante } 15 \text { minutos } \\
\text { en dias separados: } \\
\text { calor superficial } \\
\text { usando un paquete } \\
\text { de calor húmedo } \\
\left.\text { cervical ( } 77^{\circ} \mathrm{C}\right) \text {, } \\
\text { simulación usando } \\
\text { un paquete } \\
\text { húmedo cervical } \\
\text { (temperatura } \\
\text { ambiente a } \\
\text { aproximadamente } \\
24^{\circ} \mathrm{C} \text { ) y control (sin } \\
\text { tratamiento) }\end{array}$ & $\begin{array}{l}\text { Relación de activación } \\
\text { central y el torque de } \\
\text { extensión de rodilla } \\
\text { durante la contracción } \\
\text { isométrica voluntaria } \\
\text { máxima con la rodilla } \\
\text { flexionada a } 60 \text { se } \\
\text { recogieron en pre, } \\
\text { inmediatamente después, } \\
30 \text { min después y } 45 \text { min } \\
\text { después del tratamiento. } \\
\text { Temperatura de la piel del } \\
\text { cuádriceps y la rodilla y la } \\
\text { temperatura ambiente. }\end{array}$ & $\begin{array}{l}\text { No hubo interacciones significativas o efectos principales } \\
\text { en la activación central o torque de extensión de la rodilla } \\
\text { (todos } p>0.05 \text { ). } \\
\text { Los ANOVA de } 1 \text { vía revelados por la temperatura de la } \\
\text { piel y la temperatura de la piel en la rodilla durante el } \\
\text { calor superficial fue significativamente más alta que en } \\
\text { otras condiciones de tratamiento en todos los momentos } \\
(p<0,05) \text {. }\end{array}$ \\
\hline $\begin{array}{l}\text { Pietrosimo } \\
\text { ne et al. } \\
\text { (2010) } \\
\text { [16] }\end{array}$ & $\begin{array}{l}n=33 \\
\begin{array}{c}58-60 \\
\text { años }\end{array}\end{array}$ & $\begin{array}{l}\text { Tres grupos: TENS, } \\
\text { placebo y control. } \\
\text { Todos los } \\
\text { participantes } \\
\text { completaron un } \\
\text { programa } \\
\text { supervisado de } \\
\text { ejercicios pierna } \\
\text { extremidades } \\
\text { inferiores de } 4 \\
\text { semanas solo para } \\
\text { la p las } \\
\text { involucrada. } \\
\text { Se usaron TENS y } \\
\text { TENS con placebo a } \\
\text { lo largo de las } \\
\text { sesiones de } \\
\text { rehabilitación, así } \\
\text { como durante las } \\
\text { actividades diarias }\end{array}$ & $\begin{array}{l}\text { Relación de activación } \\
\text { central de cuádriceps } \\
\text { (CAR), una medida de } \\
\text { activación muscular } \\
\text { volitiva se evaluó en la } \\
\text { pierna no afectada al inicio } \\
\text { del estudio, } 2 \text { semanas y } 4 \\
\text { semanas después del inicio } \\
\text { de la intervención. }\end{array}$ & $\begin{array}{l}\text { No hubo diferencias entre los grupos para CAR }(p=0.3) \text {. } \\
\text { Aunque no se encontraron diferencias significativas, se } \\
\text { calcularon los tamaños de los efectos de los grupos } \\
\text { fuertes a moderados para el grupo TENS en } 2 \text { semanas (d } \\
=0.87 \text { ) y } 4 \text { semanas ( } d=0.54 \text { ). }\end{array}$ \\
\hline $\begin{array}{l}\text { Rice et al. } \\
\text { (2009) } \\
{[17]}\end{array}$ & $\begin{array}{l}n=16 \\
58-60 \\
\text { años }\end{array}$ & $\begin{array}{lr}\text { Dos } & \text { grupos: } \\
\text { Crioterapia } & \text { y } \\
\text { control. } & \end{array}$ & $\begin{array}{l}\text { EMG para torque pico } \\
\text { máximo del cuádriceps } \\
\text { registrador durante } \\
\text { contracciones isométricas } \\
\text { de esfuerzo máximo. }\end{array}$ & $\begin{array}{l}\text { El torque pico máximo del cuádriceps, la velocidad de } \\
\text { conducción de la fibra muscular y RMS disminuyeron } \\
\text { significativamente luego de la infusión conjunta } \\
(p<0.001) \text {. }\end{array}$ \\
\hline & & & $\begin{array}{l}\text { Luego, todos los sujetos } \\
\text { recibieron una infusión } \\
\text { conjunta experimental de } \\
\text { solución salina en la rodilla } \\
\text { a una presión intra- } \\
\text { articular de } 50 \mathrm{mmHg} \text {. }\end{array}$ & $\begin{array}{l}\text { Crioterapia condujo a un aumento significativo en el } \\
\text { torque cuádriceps y MFCV en comparación con los } \\
\text { controles }(p<0.05) \text {. } \\
\text { La diferencia en RMS no alcanzó significación estadística } \\
(p=0.13) \text {. }\end{array}$ \\
\hline $\begin{array}{l}\text { Hopkins et } \\
\text { al. }(2001) \\
\quad[18]\end{array}$ & $n=30$ & $\begin{array}{l}\text { Tres grupos: } \\
\text { Crioterapia, TENS y } \\
\text { control. }\end{array}$ & $\begin{array}{l}\text { Reflejo } \mathrm{H} \text { utilizando un } \\
\text { estímulo percutáneo para } \\
\text { el nervio femoral y }\end{array}$ & $\begin{array}{l}\text { Mediciones reflejo } \mathrm{H} \text { en la crioterapia y los grupos TENS } \\
\text { fueron mayores que las mediciones del grupo control a } \\
\text { los } 15 \text { y } 30 \text { minutos después de la inyección. }\end{array}$ \\
\hline
\end{tabular}




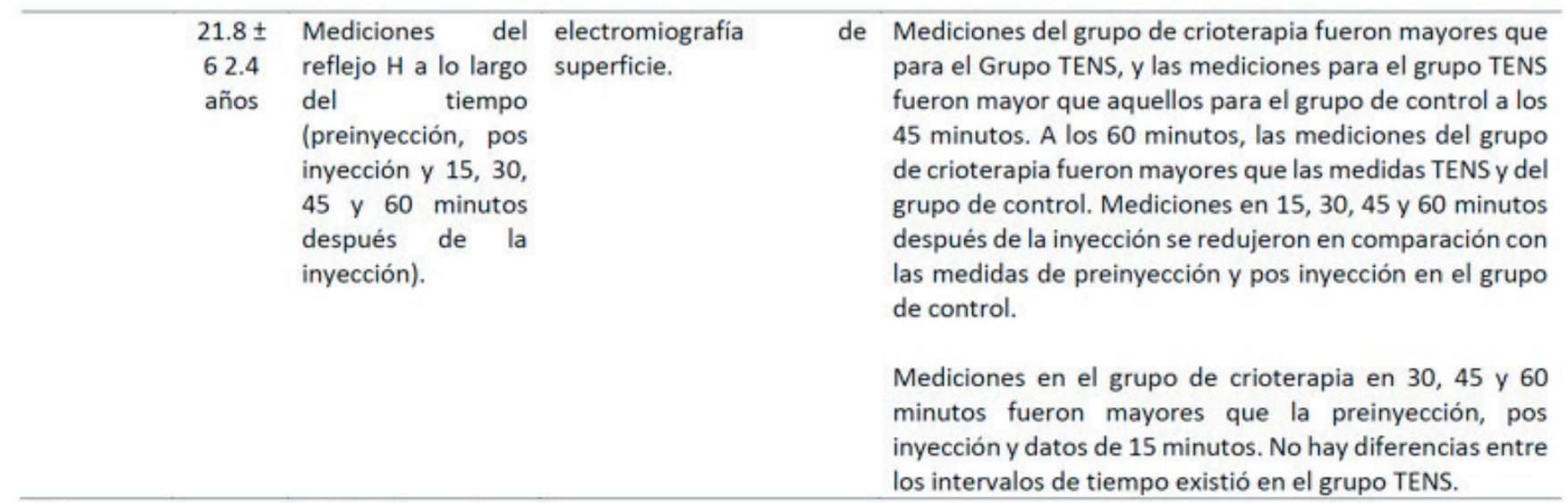

RMS: root mean square; EMG: Electromiografia;

En la Tabla 1, se relaciona la literatura revisada detallando los hallazgos significativos. En la mayoría de las publicaciones revisadas, se encontraron efectos desde un abordaje con la aplicación de medios físicos y ayudas externas (Crioterapia, TENS, Tapping) [1418] más que el abordaje del ejercicio terapéutico [12,13]. Dentro de las mediciones más utilizadas esta la EMG (Reflejo H y Onda M) $[12,14,17,18]$; mediciones con Test (SCT, TUG, 6MW, CAR, Contracciones isométricas) $[12,15,16]$; ROM [12,14]; Torque [15,17]; medición con Escalas (WOMAC, SF-12) [12] y medición de la temperatura en la piel [16]. Según las 7 publicaciones revisadas, todas reportan que la IMA incide directamente sobre el $\mathrm{MCH}$ en los pacientes con lesiones articulares, especialmente en aquellas que afectan la rodilla, comprometiendo dimensiones desde el punto de biomecánico como el torque máximo o el ROM, sin embargo, es de entender que el detrimento causado por la IMA incide directamente en la funcionalidad del individuo (como la marcha), lo que puede llevar a un mayor estudio en dicha área.

Tabla 2. Análisis de las Revisiones Sistemáticas/Meta-análisis

\begin{tabular}{|c|c|c|c|}
\hline Autor (año) & $\begin{array}{l}\text { Estudios } \\
\text { incluidos }\end{array}$ & $\begin{array}{l}\text { Sistematización de } \\
\text { Información }\end{array}$ & Resultados \\
\hline $\begin{array}{l}\text { Bartholdy et } \\
\text { al. } \\
\text { (2017) [19] }\end{array}$ & 45 & $\begin{array}{l}\text { Las intervenciones se } \\
\text { categorizaron como } \\
\text { intervenciones con ACSM o } \\
\text { intervenciones sin ACSM y se } \\
\text { compararon usando modelos } \\
\text { de metanálisis de efectos } \\
\text { aleatorios estratificados. Las } \\
\text { asociaciones entre el } \\
\text { aumento de la fuerza del } \\
\text { extensor de la rodilla y los } \\
\text { cambios en el dolor / } \\
\text { discapacidad se evaluaron } \\
\text { mediante análisis de } \\
\text { metarregresión. }\end{array}$ & $\begin{array}{l}\text { Se encontró una diferencia estadísticamente significativa a favor de las } \\
\text { intervenciones del ACSM con respecto a la fuerza del extensor de la rodilla } \\
\text { [diferencia SMD: 0,448 (IC 95\%: 0,091-0,805)]. No se observaron } \\
\text { diferencias con respecto a los efectos sobre el dolor y la discapacidad. Las } \\
\text { meta-regresiones indicaron que los aumentos en la fuerza del extensor de } \\
\text { la rodilla de 30-40\% serían necesarios para un probable efecto beneficioso } \\
\text { concomitante sobre el dolor y la discapacidad, respectivamente. }\end{array}$ \\
\hline $\begin{array}{l}\text { Harkey et al. } \\
(2014)[20]\end{array}$ & 10 & 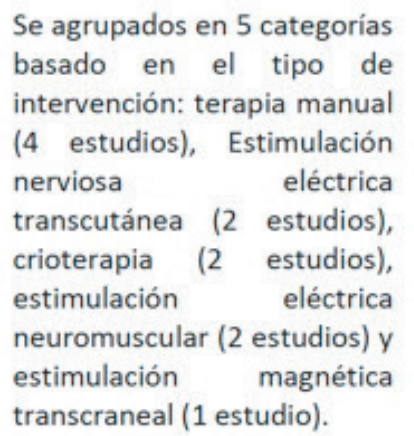 & $\begin{array}{l}\text { La estimulación nerviosa eléctrica transcutánea demostró los efectos } \\
\text { inmediatos más fuertes ( } \mathrm{d} 1 / 41.03, \mathrm{IC} 95 \%=0.06,1.92) \text { y efectos a largo } \\
\text { plazo ( } \mathrm{d}-1,93 ; \mathrm{IC} \text { del } 95 \%: 0,91 \text { a } 2,83 \text { ). Crioterapia }(\mathrm{d} 1 / 40.76 ; 95 \% \mathrm{IC}=0.13 \text {, } \\
\text { 1.59) y estimulación magnética transcraneal }(\mathrm{d}=0,54 ; \mathrm{IC} \text { del } 95 \%=0,33 \text {, } \\
1,37 \text { ) fue moderada efectos inmediatos para mejorar la activación } \\
\text { voluntaria del cuádriceps, mientras que la terapia manual ( } \mathrm{d}=0.38 ; \mathrm{IC} \text { del } \\
95 \%=0.35,1.09 \text { ) provocó solo efectos inmediatos débiles. Estimulación } \\
\text { eléctrica Neuromuscular produjo efectos positivos débiles negativos a } \\
\text { fuertes. (rango de valores d de } 1 / 40.50 \text { a } 1.87 \text { ) durante un periodo de } 3 \\
\text { semanas a } 6 \text { meses. }\end{array}$ \\
\hline
\end{tabular}




$\begin{array}{cl}\begin{array}{c}\text { Pietrosimon } \\ \text { e etal. }\end{array} & \text { Los participantes y niveles } \\ \text { (2011) [21] } & \text { medios de activación } \\ & \text { muscular se utilizaron para } \\ \text { calcular los promedios } & \text { ponderados para la } \\ & \text { extremidad afectada (14 } \\ & \text { estudios), la extremidad } \\ & \text { contralateral (de un } \\ & \text { subconjunto de } 6 \text { estudios) y } \\ & \text { un subconjunto de } \\ & \text { extremidades involucrado } \\ & \text { solo estudios que evaluaron } \\ & \text { la extremidad contralateral y } \\ & \text { población control (5 } \\ & \text { estudios). }\end{array}$

Hart et al. $18 \quad$ Un total de 21 conjuntos de (2010) [22] datos de 18 estudios fueron inicialmente identificado. Se tuvo en cuenta promedios, medidas de variabilidad y prevalencia de la falla de activación del cuádriceps (95\%) para experimentos que involucran deficiencia en LCA (10), reconstrucción de LCA (5) y dolor anterior de rodilla (3).
Medios ponderados de la extremidad involucrada $(82.2,95 \% \mathrm{Cl}=81.4$ $83.3 \%)$, extremidad contralateral $(81.7 ; \mathrm{Cl}=80.1-83.3 \%)$ y el subconjunto de extremidades involucrado $(76.8 ; \mathrm{Cl}=74.8-78.8 \%)$ se encontró que los grupos tenían activación del cuádriceps volitivo inferior comparados con los grupos de control (90; $\mathrm{Cl}=88.9-91.7 \%)$.
Datos de 3 estudios ( 1 informe de datos en papel para deficiencia en LCA y reconstrucción de LCA, 1 en dolor anterior de rodilla y el papel de postartescopia) fueron excluidos de los análisis primarios porque solo datos gráficos fueron reportados. De los 17 conjuntos de datos restantes (de 15 estudios), el promedio ponderado de control de calidad en 352 pacientes deficiencia en LCA fue $87.3 \%$ en el lado involucrado, $89.1 \%$ en el lado no involucrado, y $91 \%$ en participantes de control.

La prevalencia de falla de control de calidad varió de $0 \%$ a $100 \%$. Media Ponderada de activación del cuádriceps en 99 pacientes con reconstrucción de LCA total fue $89.2 \%$ en el lado involucrado, $84 \%$ en el lado no involucrado, y $98.5 \%$ para el grupo control, con una prevalencia que va desde $0 \%$ a $71 \%$. Treinta y ocho pacientes con dolor anterior de rodilla promediaron $\mathbf{7 8 . 6 \%}$ en el lado involucrado y el $77.7 \%$ en el lado contralateral. La falla bilateral de activación del cuádriceps se informó comúnmente en pacientes.

ECC: Ensayos Clínicos Controlados; R.S/M.A: Revisión Sistemática/Meta-análisis; LCA: Ligamento Cruzado Anterior; ACSM: American College of Sport Medicine

Para el análisis de las 4 revisiones sistemáticas/meta-análisis, se siguieron los lineamientos propuestos por la extensión PRISMA-equidad [11] para las publicaciones en salud, los cuales se detallan en la Tabla 2. En general, el análisis de estas mostró que el número de artículos que cumplieron los criterios de inclusión oscilo entre 10 hasta 45 . Los resultados fueron variados donde se encontraron diferencias estadísticamente significativas a favor de las intervenciones con ejercicio según parámetros del ACSM con respecto a la ganancia fuerza del extensor de la rodilla para un probable efecto beneficioso concomitante sobre el dolor y la discapacidad [19],

Las publicaciones que aplicaron medios físicos reportaron que el uso de TENS demostró los efectos inmediatos más fuertes a largo plazo, comparados con la Crioterapia y estimulación magnética transcraneal, mostro efectos moderados inmediatos para mejorar la activación voluntaria del cuádriceps, mientras que la terapia manual provocó solo efectos inmediatos débiles, y la NEMS mostro efectos débiles a fuertes durante un período de 3 semanas a 6 meses [20]. Con relación a la activación voluntaria del cuádriceps, la media ponderada de la extremidad lesionada y la extremidad contralateral mostraron activación del cuádriceps inferior comparados con los grupos de control [21-23].

\section{Discusión}

En la mayoría de las publicaciones revisadas, se encontraron efectos diversos en el abordaje específicamente en rodilla; posiblemente porque ésta articulación ha sido ampliamente estudiada debido a la función que cumple respondiendo a dos exigencias mecánicas opuestas: movilidad, para permitir desplazamiento del cuerpo y movimientos articulares, y estabilidad, para soportar el peso corporal, así como la carga en la fase de apoyo de la marcha [23]. Sin embargo, es de entender que el detrimento articular puede causar IMA en cualquier articulación afectando la funcionalidad del individuo, lo que puede llevar a un mayor campo de estudio en otras articulaciones del cuerpo humano. Aunque no se puede evitar la aparición de IMA (inclusive si está muy acentuada, la persona afectada puede sufrir grandes limitaciones de movimiento), se puede tratar y reducir la gravedad de la misma con una intervención fisioterapéutica adecuada. 
Se ha comprobado que la limitación en el movimiento y la consciencia de la lesión de la rodilla, obligan a la persona a reducir el movimiento o utilizar ciertas compensaciones, por miedo a sentir dolor. La IMA en la rodilla produce problemas para lograr la extensión y así evitar mayor daño articular; el problema de esta compensación es que se comenzará a abusar de la flexión de rodilla, que estará menos limitada, debilitando cada vez más el cuádriceps y sobrecargando el tendón rotuliano. Además, al perder capacidad de extensión, se compensará poco a poco con una anteversión pélvica, pues los flexores de la cadera se verán obligados a trabajar más para seguir manteniendo el movimiento.

La severidad de la IMA puede ser evaluada como inhibición del reflejo de Hoffmann (reflejo $\mathrm{H}$ ), consistente con los resultados obtenidos en este estudio. Esto tiene la ventaja que elimina los problemas de interpretación creada por dudas sobre motivación en contracciones voluntarias de los sujetos evaluados [7]. El reflejo $\mathrm{H}$ sucede por la estimulación de la fibra Ia del nervio periférico, que se provoca mecánicamente por el estiramiento del huso neuromuscular [24,25]. El reflejo $\mathrm{H}$ es parte de la expresión electrofisiológica del estiramiento (reflejo miotático); siendo un reflejo monosináptico dado por el estímulo submáximo de las fibras aferentes del nervio periférico y la respuesta motora se registra en un músculo inervado por el nervio estimulado. En el uso diario, el reflejo $\mathrm{H}$ se obtiene prácticamente solo en los músculos gemelos o sóleos gracias a la estimulación del nervio tibial posterior, y aparece en los adultos con latencia bastante estable entre 25$35 \mathrm{~m}$ [26]. La efectividad facilitadora de la retroalimentación aferente del huso neuromuscular, se controla mediante la modulación de la excitabilidad refleja segmentaria de modo que el nivel de activación muscular sea apropiado para la tarea [27]. Existen varios estudios que utilizan en reflejo $\mathrm{H}$ para analizar la IMA. Palmieri y colaboradores en 2003, evaluaron curvas de reclutamiento bilaterales para el reflejo $\mathrm{H}$ y la onda $\mathrm{M}$ del cuádriceps en lesiones de rodilla y encontraron que las relaciones de $\mathrm{H}$ máximo ( $\mathrm{Hmax}$ ) y $\mathrm{Hmax} / \mathrm{M}$ máximo (Mmax), disminuyeron la medida basal en el vasto medial del cuádriceps, mientras que no hubo cambios en el lado contralateral, demostrando disminución de la excitabilidad del conjunto de motoneuronas en la extremidad afectada [28].

Sedory y colaboradores en 2007, evaluaron la presencia de IMA en el cuádriceps e isquiotibiales en sujetos con y sin inestabilidad crónica unilateral de tobillo. El grupo de inestabilidad crónica de tobillo demostró activación central cuádriceps significativamente mayor en sus extremidades afectadas $(0.87 \pm 0.09)$, en comparación con sus extremidades no afectadas $(0.84 \pm 0.08)$, mientras que no se observó una diferencia significativa en el grupo de control. Cuando los valores de las extremidades involucradas y no involucradas se promediaron, la activación central de los isquiotibiales fue significativamente menor para el grupo de inestabilidad crónica de tobillo $(0.94 \pm 0.03)$ que para el grupo control $(0.96 \pm 0.03)$, demostrando la IMA en los músculos isquiotibiales bilateralmente y del músculo cuádriceps ipsilateral a la extremidad afectada en sujetos con inestabilidad crónica de tobillo. La excitabilidad del grupo de motoneuronas parece estar alterada en los músculos que actúan sobre las articulaciones proximales al tobillo que en aquellos con inestabilidad crónica de tobillo de manera unilateral. [29].

En la osteoartritis (OA) de rodilla, Callaghan y colaboradores en 2014, estudiaron 124 sujetos con edad promedio 55.5 \pm 7.5 años y el análisis de regresión mostro mayores niveles de IMA asociados significativamente con dolor de rodilla intenso y con menor puntuación de lesión de medula ósea [30]. Keny y colaboradores en 2014, mencionaron que las personas con OA de la rodilla muestran debilidad marcada de los músculos cuádriceps en comparación con sus controles, pero esta debilidad muscular no se debe sólo a su atrofia, sino secundaria a la IMA de los músculos periarticulares y a la disminución del volumen muscular relacionada con la edad [31].

En el caso del tobillo, Palmieri y colaboradores en 2004, determinaron si la IMA estaba presente en el sóleo, el peronéo largo y la musculatura tibial anterior después de un derrame simulado de la articulación del tobillo. Se evaluó el reflejo $\mathrm{H}$ máximo y la onda $\mathrm{M}$ máxima usando EMG de superficie después de un estímulo percutáneo al nervio ciático antes de su bifurcación en los nervios tibiales posterior y peronéo. Encontraron que las mediciones de reflejo $\mathrm{H}$ y onda $\mathrm{M}$ en todos los músculos aumentaron $(\mathrm{p} \leq 0.05)$ después del derrame articular provocado; indicando que esto puede ocurrir para estabilizar el complejo pie/tobillo con el fin de mantener postura y/o locomoción [32]. Por su parte, Klykken y colaboradores en 2011, compararon el efecto del esguince agudo de tobillo en la excitabilidad de la neurona motora de los músculos lesionados con los músculos contralaterales ilesos y los músculos de las piernas de controles sanos. Demostraron que la excitabilidad de la neurona motora del soleo en el grupo de esguinces es más alta en la extremidad lesionada (Hmax:Mmax $=0.63 ; 95 \%$ de intervalo de confianza [IC], 0.46, 0.80); el miembro lesionado (Hmax:Mmax $=0.47 ; 95 \%$ de $\mathrm{Cl}, 0,08,0,93)(\mathrm{t} 6=3,62, \mathrm{P}=$ 0,01 ). En el esguince agudo de tobillo, la excitabilidad de la neurona motora del tibial anterior tendía a ser más bajo en el tobillo lesionado (Hmax:Mmax $=0.06 ; 95 \% \mathrm{Cl}, 0.01,0.10)$ que en el tobillo ileso (Hmax:Mmax $=0.22 ; 95 \% \mathrm{Cl}, 0.09,0.35)$ [33].

Se cree entonces que el derrame articular juega un papel importante en la ocurrencia de IMA. El derrame articular de rodilla produce IMA en cuádriceps, en parte, a un mecanismo espinal presináptico. El ácido gamma-aminobutírico (GABAergicas) responsable de la inhibición presináptica de las interneuronas puede contribuir a la inhibición del cuádriceps después de una lesión contrastando hipótesis anteriores donde la IMA es causada unicamente por la activación de la interneuronas $\mathrm{Ib}$. Esto es explicable porque las terminaciones de Ruffini se estimulan cuando se produce un derrame articular, alertando y activando estructuras centrales con respecto al estado actual de la articulación. Los estímulos aferentes hacen sinapsis en las interneuronas Ib, que causan la IMA del 
cuádriceps. Aunque esta teoría aún se está comprobando, la inhibición mediada por interneuronas Ib se ha demostrado como un papel en la provocación de IMA del cuádriceps en presencia de un derrame de la articulación de la rodilla. La depresión refleja se incrementó después de la inducción del derrame experimental, lo que lleva a especular que un control regulatorio presináptico contribuyó a la disminución excitabilidad de las motoneuronas del cuádriceps. Se sabe que las vías descendentes influyen en el reflejo de activación a través de interneuronas espinales, específicamente, Interneuronas GABA-ergicas responsables de la inhibición presináptica. También se ha comprobado que la amplitud del reflejo $\mathrm{H}$ regresa al 66\% de su amplitud original dentro de 400-500 ms, que se corresponde con el curso del tiempo para la inhibición presináptica clásica [7].

La presencia de derrame articular debe dar lugar a una gran cantidad de información aferente viajando hacia el SNC, lo que permitiría a los mecanismos supraespinales hacerse cargo y regular el movimiento en la rodilla. Adicionalmente, datos que examinan la participación de la columna vertebral y vías supraespinales en presencia de una lesión o derrame en las articulaciones, debe obtenerse durante movimientos funcionales, para ver si los mecanismos de control neuronal son similares en reposo y durante la actividad. En los casos de derrame inducido, los niveles plasmáticos de catecolaminas no cambiaron después la introducción de la aguja, mostrando así que la inyección no podría ser un estresor para los voluntarios, pues no se activa el sistema nervioso simpático y el eje hipotálamo-suprarrenal-pituitaria, lo que resulta en un aumento niveles de catecolaminas sanguineos [34]. Por su parte Rice y colaboradores en 2014, demostraron que la excitabilidad corticomotora aumentó después del derrame experimental de la rodilla, posiblemente por una disminución en la inhibición del GABA-ergic dentro de la corteza motora [35], lo que se traduce en el aumento de la carga sobre la rodilla que potencialmente puede aumentar el riesgo de futuros traumatismos o degeneración de la articulación [36].

Aunque no existe hoy en día un tratamiento eficaz para la IMA, el protocolo de rehabilitación fisioterapéutica después de la lesión articular debe centrarse en el control neuromuscular y alteraciones biomecánicas presentes como resultado del daño. Para realizar acciones efectivas, el fisioterapeuta debe comprender y evaluar los mecanismos que intervienen en la generación, modulación y perpetuación del dolor como base de una intervención terapéutica integral [37], para ello, la Fisioterapia tiene un papel importante en el tratamiento de la IMA, empleando modalidades de tratamiento como medios físicos, electroterapéuticos y ejercicio, los cuales en muchos casos pueden ser alternativas óptimas para su tratamiento [38].

Algunas de estas modalidades son:

- Estimulación Eléctrica Neuromuscular (NMES): Este tipo de corriente activa directamente al músculo, por lo que se salta las vías inhibitorias y minimiza la atrofia muscular. Según Rafsanjani y colaboradores en 2017, la estimulación eléctrica del nervio peronéo común concurrente con el esfuerzo voluntario máximo puede eliminar la IMA del cuádriceps en OA de rodilla [39] siendo beneficiosa para la rehabilitación. Además, los beneficios del NMES parecen ser dependientes de la dosis, con alta intensidad, pues estimulaciones máximas toleradas por el paciente, prueban ser más efectivas que aquellas a intensidades más bajas [40]. Por el contrario, un ensayo controlado aleatorizado recientemente informó que no hay diferencia en el cambio en la fuerza del cuádriceps y activación central de 3-12 meses después de reemplazo de rodilla en un grupo que se sometió únicamente a entrenamiento de resistencia en comparación con un grupo que experimentó entrenamiento de resistencia combinado con NMES [41].

- Estimulación Magnética Transcraneal (EMT): Si bien la activación de la corteza motora primaria se ha implicado como un factor clave en la IMA después de la lesión de la articulación de la rodilla, no se ha desarrollado un protocolo de rehabilitación. Según Lee y colaboradores en 2017, los sujetos que recibieron EMT mostraron niveles significativamente más altos de contracción muscular voluntaria después del entrenamiento y el efecto beneficioso de la estimulación aumentó a medida que avanzaba la rehabilitación. Aunque la EMT en sí misma no mejora directamente los síntomas relacionados con las lesiones de rodilla, el uso de esta técnica puede proporcionar una ventana de tiempo para una intervención efectiva al disipar el efecto no deseado de la IMA durante la rehabilitación fisioterapéutica [42].

- Estimulación Nerviosa Eléctrica Transcutánea (TENS): Es una estimulación nerviosa transcutánea que hace descender la pérdida de activación por el reflejo $\mathrm{H}$. Ha mostrado ser útil en casos de artrosis, que suelen provocar rigidez articular. Según Harkey y colaboradores en 2014, el TENS demostró efectos fuertes y consistentes en el aumento de la activación voluntaria del cuádriceps y puede ser la mejor intervención desinhibidora para mejorar la IMA [43]. Igualmente Son y colaboradores en 2016, demostraron que el TENS a nivel sensorial, restauró significativamente la función motora del cuádriceps inhibida en comparación con el tratamiento placebo $(\mathrm{p}<0,05)$ y hubo una correlación negativa entre los cambios en la contracción voluntaria máxima y el dolor de rodilla $(\mathrm{r}=0.33, \mathrm{p}<0.001)$, y la activación central y el dolor de rodilla $(\mathrm{r}=0.62, \mathrm{p}<0.001)$ [44]. Hopkins y colaboradores en 2002, encontraron que el TENS a alta frecuencia $(120 \mathrm{~Hz}$, ancho de pulso 0.1 segundos) previene la disminución en la amplitud del reflejo $\mathrm{H}$ del cuádriceps después de la infusión de fluido en la articulación de la rodilla [17].

- Crioterapia: La aplicación de hielo o técnicas de crioterapia, disminuyen la sensación de dolor y la inflamación. Según Gabler y colaboradores en 2016, tanto la crioterapia y el TENS afectan positivamente el control de calidad voluntario al desinhibir las motoneuronas del cuádriceps. En esencia, estas modalidades 
proporcionan estímulos aferentes exitatorios a la médula espinal, lo que anula la señal inhibitoria aferente que surge de la articulación afectada $[17,45]$. Sandoval y colaboradores en 2011, demostraron que las aplicaciones de tres modalidades de crioterapia redujeron la temperatura de la piel (media 18.2 ${ }^{\circ} \mathrm{C} ; \mathrm{p} \leq 0.001$ ), igualmente disminuyo la amplitud, la latencia y la duración del potencial de acción compuesto $(\mathrm{p}<0.05)$. El masaje con hielo, paquete de hielo y la inmersión en agua fría redujeron la velocidad de conducción del nervio sensorial por $20.4,16.7$ y $22.6 \mathrm{~m} / \mathrm{s}$; mismas modalidades redujeron la velocidad de conducción del nervio motor en $2.5,2.1$ y $8.3 \mathrm{~m} / \mathrm{s}$, respectivamente [46].

- Ejercicio: La pérdida de fuerza es común en la IMA y en el caso de la rodilla, se ha demostrado que los déficits inmediatos de activación del cuádriceps después del derrame articular resultan en alteraciones en la fuerza muscular, el momento extensor y la fuerza de reacción vertical del suelo durante la marcha [47]. La influencia de la mejoría de la actividad muscular en la carga articular de rodilla, optimiza la función muscular y propioceptiva observados en personas con IMA [48], por ende, la prescripción de ejercicio con fines de rehabilitación logra reducir el dolor y mejora la funcionalidad [49]. Blackburn y colaboradores en 2014, demostraron que el fortalecimiento de los cuádriceps mediante vibración corporal total o vibración muscular local, mejoran la función muscular después de la patología inducida en la rodilla, minimizando de manera efectiva el IMA [50]. También Hendy y colaboradores en 2015, reportaron efectos positivos con la combinación de ejercicio y estimulación transcraneal de corriente continua con ánodo para la mejoría de la fuerza, debido a aumentos significativos en la excitabilidad corticomotoneuronal y disminuciones en la inhibición intracortical de intervalo corto [51]. Hart y colaboradores en 2014, demostraron que después de la reconstrucción del LCA, los pacientes con IMA quien realizaron ejercicios de rehabilitación inmediatamente después de la crioterapia experimentaron mayores ganancias de fuerza que aquellos que realizaron crioterapia o ejercicios solos. [52]. Regímenes de ejercicio que enfatizan la contracción muscular fuerte y herramientas clínicas que facilitan la activación muscular como la retroalimentación biológica y la estimulación eléctrica neuromuscular pueden ser necesarias para revertir la falla de activación del cuádriceps y la IMA [53]. La fuerza muscular es una función física relevante y el aumento de la misma es un objetivo clínico en los pacientes con lesión articular. La evidencia emergente con respecto a las estrategias de rehabilitación que utiliza modalidades desinhibitorias para mejorar la activación neuromuscular en conjunto con el fortalecimiento muscular tradicional, tienen por objetivo, maximizar las ganancias de fuerza en la articulación comprometida [54]. Las intervenciones de ejercicio siguiendo los criterios del ACSM para el entrenamiento de la fuerza proporcionan resultados superiores en la fuerza del extensor de la rodilla. Un aumento de menos del $30 \%$ en la fuerza del cuádriceps probablemente no sea clínicamente beneficioso en términos de cambios en el dolor y la discapacidad [17].
- Medicamentos: Aunque no son prescritos directamente por el fisioterapeuta, se han reportado efectos positivos con el uso de medicamentos y procedimientos médicos con fines analgésicos. Entre estos están la aspiración de líquido de la articulación, inyección intra-articular de corticosteroides, medicamentos anti-inflamatorios no esteroideos (AINES), Sulfato de glucosamina y anestesia local [55].

La IMA sigue siendo una barrera importante para la rehabilitación efectiva en pacientes con una lesión y/o cirugía articular; por lo tanto, una mejor comprensión de los mecanismos subyacentes permitirá al fisioterapeuta el desarrollo de estrategias terapéuticas mejorando la rehabilitación de pacientes con patología articular [3]. En general, este articulo muestra efectos desde un abordaje con la aplicación de medios físicos y ejercicio terapéutico, estas intervenciones comunes en el actuar del fisioterapeuta. Según las 4 revisiones sistemáticas/meta-análisis, las intervenciones con ejercicio según los parámetros del ACSM promueven la ganancia fuerza del cuádriceps. La aplicación de medios físicos como el TENS, la Crioterapia y la EMT, mejoran la activación voluntaria del cuádriceps [20]. Es por ello que se hace necesario un conocimiento a fondo de las implicaciones de la IMA sobre la funcionalidad, siendo esta premisa un reto que debe seguir profundizándose e investigándose para dar mayor soporte basado en la evidencia científica. Quizás el beneficio más relevante de la Fisioterapia, se basa en la mejoría funcional del individuo; pero para lograrlo hay que mejorar la calidad de las investigaciones para facilitar evidencia que sustenten las diferentes modalidades de intervención del fisioterapeuta como estudioso del $\mathrm{MCH}$.

\section{Agradecimientos:}

El autor agradece a la Universidad Manuela Beltrán por el apoyo en la elaboración del manuscrito.

Conflictos de interés: El autor no declara conflictos de intereses

\section{Referencias}

1. Morales-Osorio MA. Del Modelo Biomédico al Modelo Biopsicosocial: El desafío pendiente para la fisioterapia en el dolor músculoesquelético crónico. Rev Fac Ciencias Salud UDES. 2016;3(2):97-101.

2. Sánchez Y, Matiz M, Mora L, Santander C, Ramírez C. Rehabilitación en la inhibición muscular artrogénica: Revisión Sistemática. Rev Col Reh. 2017;16(2):16-23.

3. Rice, D. McNair, P. Quadriceps Arthrogenic Muscle Inhibition: Neural Mechanisms and Treatment Perspectives. Semin Arthritis Rheum. 2010;40(3):250-266.

4. Hopkins JT, Christopher D. Arthrogenic Muscle Inhibition: A limiting factor in joint rehabilitation. J Sport Rehab. 2000;9(2):135-159. 
5. Young A. Current issues in arthrogenous inhibition. Ann Rheum Dis. 1993;52:829-834.

6. IASP. Pain terms: a current list with definitions and notes on usage. Pain. 1986; Suplemento 3:S215-S221.

7. Zegarra Piérola JW. Bases fisiopatológicas del dolor. Acta Med Per. 2007;24(2):105-108.

8. Palmieri RM, Weltman A, Edwards JE, Tom JA, Saliba EN, Mistry DJ, Ingersoll CD. Pre-synaptic modulation of quadriceps arthrogenic muscle inhibition. Knee Surgery, Sports Traumatology, Arthroscopy. 2005;13(5):370-376.

9. Suter E, Herzog W. Does muscle inhibition after knee injury increase the risk of osteoarthritis? Exerc Sport Sci Rev. 2000;28(1):15-18.

10. Palmieri RM, Ingersoll CD, Edwards JE, Hoffman MA, Stone MB, Babington JP, Cordova ML, Krause BA. Arthrogenic muscle inhibition is not present in the limb contralateral to a simulated knee joint effusion. Am J Phys Med Rehabil. 2003;82(12):910-916.

11. Welch V, Petticrew M, Tugwell P, Moher D, O’Neill J, et al. PRISMA-Equity 2012 Extension: Reporting Guidelines for Systematic Reviews with a Focus on Health Equity. Rev Pan Salud Publica. 2013;34(1):60-67.

12. Bade MJ, Struessel T, Dayton M, Foran J, Kim RH, Miner T, et al. Early High-Intensity Versus Low-Intensity Rehabilitation After Total Knee Arthroplasty: A Randomized Controlled Trial. Arthritis Care Res (Hoboken). 2017;69(9):1360-1368.

13. Walsh R, Kinsella S. The effects of caudal mobilization with movement (MWM) and caudal self-mobilization with movement (SMWM) in relation to restricted internal rotation in the hip: A randomized control pilot study. Man Ther. 2016;22:9-15.

14. Chou E, Kim K-M, Baker AG, Hertel J, Hart JM. Lower leg neuromuscular changes following fibular reposition taping in individuals with chronic ankle instability. Man Ther. 2013;18(4):316-320.

15. Warner B, Kim KM, Hart JM, Saliba S. Lack of effect of superficial heat to the knee on quadriceps function in individuals with quadriceps inhibition. J Sport Rehabil. 2013;22(2):93-99.

16. Pietrosimone BG, Saliba SA, Hart JM, Hertel J, Ingersoll $\mathrm{CD}$. Contralateral effects of disinhibitory TENS on quadriceps function in people with knee osteoarthritis following unilateral treatment. N Am J Sports Phys Ther. 2010;5(3):111-121.

17. Rice D, McNair PJ, Dalbeth N. Effects of Cryotherapy on Arthrogenic Muscle Inhibition Using an Experimental Model of Knee Swelling. Arthritis \& Rheumatism. 2009;61(1):78-83.

18. Hopkins JT, Ingersoll CD, Edwards J, Klootwyk JE. Cryotherapy and Transcutaneous Electric Neuromuscular Stimulation decrease Arthrogenic Muscle Inhibition of the Vastus medialis after Knee Joint Effusion. J Athl Train. 2001;37(1):25-31.

19. Bartholdy C, Juhl C, Christensen R, Lund H, Zhan W, Henriksen $M$. The role of muscle strengthening in exercise therapy for knee osteoarthritis: A systematic review and metaregression analysis of randomized trials. Semin Arthritis Rheum. 2017;47(1):9-21.
20. Harkey MS, Gribble PA, Pietrosimone BG. Disinhibitory Interventions and Voluntary Quadriceps Activation: A Systematic Review. J Athl Train. 2014;49(3):411-421.

21. Pietrosimone BG, Hertel J, Ingersoll CD, Hart JM, Saliba SA. Voluntary Quadriceps Activation Deficits in Patients with Tibiofemoral Osteoarthritis: A Meta-Analysis. PM\&R Journal. 2011;3(2):153-162.

22. Hart JM, Pietrosimone BG, Hertel J, Ingersoll CD. Quadriceps Activation Following Knee Injuries: A Systematic Review. Journal of Athletic Training. 2010;45(1):87-97.

23. Flandry F, Hommel G. Normal Anatomy and Biomechanics of the Knee. Sports Med Arthrosc Rev. 2011;19:82-92.

24. Trimble MH, Du P, Brunt D, Thompson FJ. Modulation of triceps surae $\mathrm{H}$-reflexes as a function of the reflex activation history during standing and stepping. Brain Res. 2000;858(2):274-283.

25. Estañol B, Montes de Oca-Delgado L, Senties-Madrid H, Baizabal-Carballo F, López-García L, García-Ramos G. El reflejo $\mathrm{H}$ del músculo tibial anterior puede ser obtenido con facilidad. Arch Neurocien (Mex). 2006;11(4):241-244.

26. Sabater Hernández H, González Roig JL, Acuña Barbón LM. Utilidad del reflejo $\mathrm{H}$ en la evaluación de la espasticidad. Rev Cubana Med Fís Rehab. 2010;2(1):34-40.

27. Pérez-Parra JE, Henao-Lema CP. Efecto de la movilización articular sobre la amplitud del Reflejo $\mathrm{H}$ en personas con espasticidad. Rev. Cienc. Salud. 2011;9(2):125-140.

28. Palmieri RM, Ingersoll CD, Edwards JE, Hoffman MA, Stone MB, Babington JP, Cordova ML, Krause BA: Arthrogenic muscle inhibition is not present in the limb contralateral to a simulated knee joint effusion. Am J Phys Med Rehabil. 2003;82:910-916.

29. Sedory EJ, McVey ED, Cross KM, Ingersoll CD, Hertel J. Arthrogenic Muscle Response of the Quadriceps and Hamstrings with Chronic Ankle Instability. J Athl Train. 2007;42(3):355-360.

30. Callaghan MJ, Parkes MJ, Hutchinson CE, Felson DT. Factors associated with arthrogenous muscle inhibition in patellofemoral osteoarthritis. Osteoarthritis Cartilage. 2014;22(2014):742-746.

31. Keny MS, Sadekar VN, Bandekar SM. Osteoarthritis of the Knee-Need for Risk Factor Modification. RRJMHS. 2014;3(4):1-10.

32. Palmieri RM, Ingersoll CD, Hoffman MA, Cordova ML, Porter DA, Edwards JE, et al. Arthrogenic muscle response to a simulated ankle joint effusion. Br J Sports Med. 2004;38:26-30. 33. Klykken LW, Pietrosimone BG, Kim KM, Ingersoll CD, Hertel J. Motor-Neuron Pool Excitability of the Lower Leg Muscles After Acute Lateral Ankle Sprain. J Athl Train. 2011;46(3):263-269.

34. Palmieri RM, Weltman A, Edwards JE, Tom JA, Saliba EN, Mistry DJ, Ingersoll CD. Pre-synaptic modulation of quadriceps arthrogenic muscle inhibition. Knee Surg Sports Traumatol Arthrosc. 2005;13:370-376.

35. Rice DA, McNair PJ, Lewis GN, Dalbeth N. Quadriceps arthrogenic muscle inhibition: the effects of experimental knee joint effusion on motor cortex excitability. Arthritis Res Ther. 2014;16:502-509. 
36. Palmieri-Smith RM, Kreinbrink J, Ashton-Miller JA, Wojtys EM. Quadriceps Inhibition Induced by an Experimental Knee Joint Effusion Affects Knee Joint Mechanics During a SingleLegged Drop Landing. Am. J. Sports Med. 2007;35:1269-1277. 37. Moretti FA, Ezequie, R, Marvulle V, Pollak DF, Riera R. Assessing knowledge on Fibromyalgia among internet users. Revista Brasileira Reumatologia. 2011;51(1):7-19.

38. Pinzón ID. Actualización en fibromialgia: implicaciones neurofisiológicas y biomecánicas útiles para el abordaje fisioterapéutico. Rev. Col. Reh. 2014;13:96-102.

39. Rafsanjani H, Khademi-Kalantari K, Rezasoltani A, Naimi SS, Ghasemi M, Jaberzadeh S. Immediate effect of common peroneal nerve electrical stimulation on quadriceps muscle arthrogenic inhibition in patients with knee osteoarthritis. J Bodyw Mov Ther. 2017;21(4):879-883.

40. Fitzgerald GK, PivaSR, Irrgang JJ. A modified neuromuscular electrical stimulation protocol for quadriceps strength training following anterior cruciate ligament reconstruction. J Orthop Sports Phys Ther. 2003;33(9):492-501.

41. Petterson SC, Mizner RL, Stevens JE, Raisis L, Bodenstab A, Newcomb W, Snyder-Mackler L. Improved function from progressive strengthening interventions after total knee arthroplasty: a randomized clinical trial with an imbedded prospective cohort. Arthritis Rheum. 2009;61(2):174-183.

42. Lee JM, Lee JH. Benefits of using transcranial magnetic stimulation as a tool to facilitate the chronic knee injury rehabilitation. J. Phys. Ther. Sci. 2017;29:733-736.

43. Harkey MS, Gribble PA, Pietrosimone BG. Disinhibitory interventions and voluntary quadriceps activation: a systematic review. J Athl Train. 2014;49(3):411-421.

44. Son SJ, Kim H, Seeley MK, Feland JB, Hopkins JT. Effects of transcutaneous electrical nerve stimulation on quadriceps function in individuals with experimental knee pain. Scand J Med Sci Sports. 2016;26(9):1080-1090.

45. Gabler CM, Lepley AS, Uhl TL, Mattacola CG. Comparison of Transcutaneous Electrical Nerve Stimulation and Cryotherapy for increasing Quadriceps activation in patients with Knee Pathologies. J Sport Rehabil. 2016;25(3):294-300.

46. Sandoval MC, Herrera E, Camargo DM. Efecto de tres modalidades de crioterapia sobre la temperatura de la piel durante las fases de enfriamiento y recalentamiento. Salud UIS. 2011;43(2):119-129.

47. Pietrosimone B, Lepley AS, Murray AM, Thomas AC, Bahhur NO, Schwartz TA. Changes in voluntary quadriceps activation predict changes in muscle strength and gait biomechanics following knee joint effusion. Clin Biomech (Bristol, Avon). 2014;29(8):923-929.

48. Machner A, Pap G, Awiszus F. Evaluation of quadriceps strength and voluntary activation after unicompartmental arthroplasty for medial osteoarthritis of the knee. J Orthop Res. 2002;20(2002):108-111.

49. Bennell KL, Wrigley TV, Hunt MA, Lim BW, Hinman RS. Update on the role of muscle in the genesis and management of knee osteoarthritis. Rheum Dis Clin North Am. 2013;39(1):145176.
50. Blackburn JT, Pamukoff DN, Sakr M, Vaughan AJ, Berkoff DJ. Whole body and local muscle vibration reduce artificially induced quadriceps arthrogenic inhibition. Arch Phys Med Rehabil. 2014;95(11):2021-2028.

51. Hendy AM, Teo WP, Kidgell DJ. Anodal Transcranial Direct Current Stimulation Prolongs the Cross-education of Strength and Corticomotor Plasticity. Med Sci Sports Exerc. 2015;47(9):1788-1797.

52. Hart JM, Kuenze CM, Diduch DR, Ingersoll CD. Quadriceps Muscle Function After Rehabilitation with Cryotherapy in Patients with Anterior Cruciate Ligament Reconstruction. J Athl Train. 2014;49(6):733-739.

53. Stevens JE, Mizner RL, Snyder-Mackler L. Quadriceps strength and volitional activation before and after total knee arthroplasty for osteoarthritis. J Orthop Res. 2003;21(2003):775-779.

54. Pietrosimone B, Blackburn JT, Harkey MS, Luc BA, Pamukoff DN, Hart JM. Clinical strategies for addressing muscle weakness following knee injury. Clin Sports Med. 2015;34(2):285-300.

55. Rice DA. Quadriceps arthrogenic muscle inhibition: Neurophysiological mechanisms and possible therapeutic interventions. AUT University. Thesis for the degree of Doctor of Philosophy (PhD). 2012. 